\title{
OPERATING COSTS OF AGRICULTURAL HOLDINGS WITH EQUAL PRODUCTION POSSIBILITIES
}

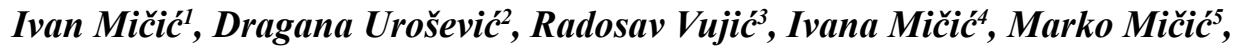 \\ Marija Mičić ${ }^{6}$
}

\begin{abstract}
Research subject is the analysis of state and basic problems in farm production of pork, as well as economic analysis of fattening pigs. Research included specific case studies on a family farm, pig farm in Gračanica, found in central Kosovo and Metohija and a cooperative farm, a pig farm in Žitorađa located in Toplica district. During a time period in 2012, the volume and applied technology of producing fattened pigs was monitored on both farms and given economic results were analyzed. In the observed period it was determined that the number of fattened livestock on the private farm was 40, while on the cooperative farm it was 30,000. Produced fattened pig on the private farm costs 87 $€$ and pork meat side is $1.16 € / \mathrm{kg}$. The price of a fattened pig on a cooperative farm is $142 €$ and pork meat side is $1.94 € / \mathrm{kg}$. Average weight of a fattened pig on both farms is $100 \mathrm{~kg}$, while the share of pork meat sides varies from $78 \%-79 \%$.
\end{abstract}

Key words: Production offattened pigs, pork meat-pork sides, price, quality, economic results.

JEL: $Q 12, Q 13$

1 Ivan Mičić M.A., PhD student, University in Belgrade, Faculty of Agriculture, Nemanjina street no. 6, 11080 Zemun, Srbija, Phone:+381 1126153 15, E-mail: divanlav@gmail.com

2 DraganaUrošević M.A., University in Belgrade, Faculty of Agriculture, Nemanjina street no. 6, 11080 Zemun, Srbija, Phone: +381 63881 49 35, E-mail: dragana.urosevic91@yahoo.com

3 Radosav Vujić, P.hD., Agricultural expert and extension service Valjevo, Birčaninova street no. 128A, 14000 Valjevo, E-mail: pssvaljevo@mts.rs

4 IvanaMičić, M.A, PhD student, Facultyof Economics, University of Niš, Trgkralja Aleksandra Ujedinitelja 11 Niš,Srbija, Phone: +381 63233 603, E-mail: ivancica@gmail.com

5 Marko Mičić, Dipl. ecc., Facultyof Economics, University of Niš, Trgkralja Aleksandra Ujedinitelja 11 Niš,Srbija, Phone: +381 63680 040, E-mail: markomicic89@,gmail.com

6 MarijaMičić, M.A, PhD student, Faculty of Technology in Leskovac, University of Niš, BulevarOslobođenja 124 Leskovac, Serbia, Phone: +381 6286745 98, E-mail: marija84micic@gmail.com

EP 2016 (63) 2 (407-428) 


\section{Introduction}

Production of pork meatis one of activities in agriculture which secures a source of income to all participants in the production chain, conducted in several technological, mutually organization-dependent phases (from care, breeding and feeding of sows and gilts, farrowing, upbringing of piglets and fattening, through feeding, etc.). Regardless of what the natural indicators in intensive, market oriented production of pigs are, it is very difficult to provide a detailed insight into the production cost of fattened pigs, which represents the research basis of the paper and proof that the pig production process is cost effective. Tešanović (1969) determines that with an increase in the number of piglets per sow from 10 to 20 , the production cost per piglet reduced by $79.09 \%$, while cost per sow increased by $11.67 \%$ per annum. Increase in the genetic basis of pigs represents a necessary precondition for the achievement of greater intensity in this branch of livestock production. So Vidović et al. (2012) report that the annual genetic progress for average daily growth was 8-11 gr, food conversion from $0.03-0.05 \mathrm{~kg}$ as well as $0.35-1.00 \%$ for the content of meat in sides. Based on previous research and results in practice there is opinion that better results can be expected in due time as follows: 30 fattened pigs per sow per year, conversion of food bellow $2 \mathrm{~kg}$, less than 120 days of life to reach $100 \mathrm{~kg}$ of body weight, daily gain of live weight of about $2 \mathrm{~kg}$. Rahelić (1984) states that the characteristic of breeds of pigs as well as individual characteristics of livestock to achieve greater daily weight gain, greater amount of meat and better carcass yield in same growing conditions are of great significance to successful and cost-effective pork meat production. By better use of these properties the fattening period is reduced and at the same time total production is increased. Živković, Perunović, (2012) state that pork meat production in Serbia is characterized by the increasing participation of large farms (10,000 to 30,000, and more fattened pigs per year), and quality of pigs has significantly increased, especially on farms, and it can be said it is approaching the European average. Pork meat production is carried out by determining production cost of $1 \mathrm{~kg}$, produced pork meat sides in first and second phase. Research of the economic parameters of producing fattened pigs deals with costs in the first phase of the production process and determining total cost of producing pork meat sides in the second phase, by the division calculation method. Tomović et al. (2005) state that there is a possibility of using the two point method for grading pork meat sides in slaughterhouses whose weekly capacity is less that 200 pigs. Orović et al. (2015) analyze in their paper the business of three groups of 20 individual farms with different primary production, crop, fruit growing and livestock production. Necessary data for production of a basic model was collected by a survey on 60 agricultural holdings on the territory of Toplica. Research in the paper has the goal to provide wither application with the given results in relation to both farms. This allows for the given results to have common, rather than only local significance (Andrić, 1998; Jovanović et al., 1998). 


\section{Research goal}

This paper represents data given by research results, both during research and procedure analysis. During research and proving, primarily a scientific method is used, whose basic application enables explanation and prediction of relations between individual relevant inputs, and results of achieved effects in production of pork meat. In accordance with the development strategy of producing pork meat, the research goal is to improve the quality of pork meat production which originate from noble pure breed, raised on farms A and B, by a large number of analyzed and realized economic parameters. The representation of the given results of plant production on farm A and pig production on both farms will be given. During preparation of this work, data from multiple sources was used. The data used were the production quantity, analysis of pig production and pork meat in long time period. The analysis of this data wouldn't be possible if it weren't approved by both farms and the data was processed by mathematical-statistical methods. This data source was especially important when determining average values, loss, food conversion etc.

\section{Materials and methods}

The research conducted included family agricultural holding on farm A in Gračanica whose owner is Bojan Jovanović, address Kosovske Devojke 417 and a cooperative farm B, "1 Decembar" in Žitorađa. Both farms have a closed production cycle that includes pig production fattening. Farm A produces 40 fattened pigs and farm $\mathrm{B}$ around 30,000 a year. Within farm A 2 persons and 4 minors are employed and in farm B 80 people with corresponding qualifications are employed. Costs of producing fattened pigs on both farms are based on natural indicators determined based on research done in 2012 and all variable categories of costs in accordance with the production process. Material costs relate to consumption of nutrients and medicine used in the production process. Amortization costs are covering 2012 based on norms of necessary space and equipment, we approach the investment estimate, estimate of amortization costs based on which the fixed costs categories are calculated (Šegrt, Kolarski, 2015). When determining costs of producing costs we start from the price of a pig, weight of a pork meat side obtained by slaughter, variable costs of slaughter service and freezing. Results of plant production of farm A and fattened pig production on both farms relates to one year period (2012). In order to make a conclusion, the production parameters were monitored: grain on farm A and food consumption of farms A and B per $1 \mathrm{~kg}$ of growth, total growth and cost of food on both farms:

Total food consumption

food consumption per kilogram of growth $=$

Total growth

Total growth $=$ number of feeding days $\mathrm{x}$ growth per feeding day

Total revenue

production efficiency $=$

Total expenses

EP 2016 (63) 2 (407-428) 


production profitability $=\frac{\text { Gain }}{\text { Investment }} \mathrm{h} 100$
Profit rate (profitability) $=\frac{\text { Gain }}{\text { Total revenue }} \mathrm{h} 100$

Significance of results in production of fattened pigs and pork meatin 2012 was followed independently on farms A and B during one research year.

\section{Research results and discussion}

Starting from the previously pointed facts and characteristics of pork meat side production strategy, we analyzed on both farms:

- $\quad$ Plant production on 10 ha on farm A,

- $\quad$ Pork meat side production on farms A and B,

- Characteristics of pork meat side quality on both farms.

We analyzed in the paper:

1. Production of meatwith special emphasis on pork meat production on the province Kosovo and Metohija in Gračanica on a pig farm A of the owner Bojan Jovanović, Kosovke Devojke street no. 417 and a cooperative farm B "1 Decembar" in Žitorađa in Toplica county in Serbia.

2. Production efficiency of pork meaton farms A and B by using achieved economic indicators of production.

Next to theoretical explanation and application on general examples, we showed efficiency of optimal feeding on an example of feeding mixture for fattened pigs on farm A. Production costs of fattened pigs on both farms are based on naturally determined indicators. Calculation of fixed and variable costs was calculated on both farms in accordance with the production process. Also costs are related to consumption of nutrients and medicine which are used in the production process, as well as amortization of the livestock, existing space and equipment which is done based on norms.

Natural and financial indicators of agricultural production on the farm shown in 2012 were: corn (Table1), triticale (Table2), wheat (Table3)and barley (Table4). 
Table 1. Achieved economic indicators of corn production on 2 ha

\begin{tabular}{|c|c|c|c|c|c|c|}
\hline No. & Production year: 2012 & Amount & $\begin{array}{c}\text { Unit of } \\
\text { measure }\end{array}$ & Price & $\begin{array}{c}\text { Unit of } \\
\text { measure }\end{array}$ & $\begin{array}{c}\text { Amount of EUR } \\
\text { on } 2 \text { ha }\end{array}$ \\
\hline $\mathbf{I}$ & Revenue & & & & & \\
\hline 1. & Corn from 2 ha & 6.5 & $\mathrm{t} / \mathrm{ha}$ & 130.00 & $\mathrm{EUR} / \mathrm{t}$ & 1,690.00 EUR \\
\hline 2. & Cornstalks from 2 ha & 9 & t/ha & 17.98 & $\mathrm{EUR} / \mathrm{t}$ & 323.70 EUR \\
\hline A) & Total revenue (1 to 2) for2 ha & & & & & 2,013.70 EUR \\
\hline 3 & Expenses & & & & & \\
\hline 4. & Seed for 2 ha & 50 & $\mathrm{~kg}$ & 1.50 & $\mathrm{EUR} / \mathrm{kg}$ & 75.00 EUR \\
\hline 5. & Fertilizer & & & & & \\
\hline 6. & Manure for 2 ha & 10 & $\mathrm{t}$ & 4.00 & $\mathrm{EUR} / \mathrm{t}$ & 40.00 EUR \\
\hline 7. & KAN $(29 \% \mathrm{~N})$ & 400 & $\mathrm{~kg}$ & 0.30 & EUR/kg & 120.00 EUR \\
\hline 8. & Pesticides & & & & & \\
\hline 9. & Guardian & 6 & $\mathrm{~L}$ & 3.00 & EUR/L & 18.00 EUR \\
\hline 10. & Tezis & 6 & $\mathrm{~L}$ & 2.50 & EUR/L & 15.00 EUR \\
\hline 11. & Irrigation & & & & & \\
\hline 12. & Energy source for2 ha & 15 & $\mathrm{~L}$ & 1.40 & EUR/L & 42.00 EUR \\
\hline 13. & Diesel fuel & 60 & $\mathrm{~L}$ & 1.40 & EUR/L & 84.00 EUR \\
\hline 14. & Maintaining mechanics & 2 & ha & 15.00 & EUR/ha & 30.00 EUR \\
\hline 15. & Paid services & & & & & \\
\hline 16. & Plowing & 2 & ha & & EUR/ha & 0 EUR \\
\hline 17. & Land preparation & 2 & ha & & EUR/ha & 0 EUR \\
\hline 18. & Sowing & 2 & ha & 35.00 & EUR/ha & $70.00 \mathrm{EUR}$ \\
\hline 19. & Harvest & 2 & ha & 65.00 & EUR/ha & $130.00 \mathrm{EUR}$ \\
\hline 20. & Paid seasonal work force & 40 & $\begin{array}{l}\text { working } \\
\text { hours }\end{array}$ & 1.50 & $\begin{array}{c}\text { EUR/ } \\
\text { working } \\
\text { hour }\end{array}$ & 60.00 EUR \\
\hline 21. & Other variable costs & & & & & \\
\hline B) & Total costs(3 to 21) & & & & & 684.00 EUR \\
\hline II & GAIN/LOSS & & & & & \\
\hline 22. & Total without incentive $(\mathrm{A}-\mathrm{B})$ & & & & & 1,329.70 EUR \\
\hline 23. & Per ha without incentive $(22: 17)$ & & & & & 664.85 EUR \\
\hline 24. & Costs of grain in $\mathrm{kg}(22: 1)$ & & & & & 0.10 EUR \\
\hline 25. & Production efficiency (A : B) & & & & & 2.94 \\
\hline 26. & Revenue profitability (22:A)h100 & & & & & $66.03 \%$ \\
\hline
\end{tabular}

Source: Authors'calculation based on data from Mičić, 2012

From Table 1 we can see that the average corn yield on the farm in 2012 was $6.5 \mathrm{t} /$ ha and in the observed period in 2912 moved in the span from 6.0 t/ha to $7.0 \mathrm{t} / \mathrm{ha}$. Total realized profit on 2 ha is $1,329.70 €$, production efficiency is 2.94 and revenue profitability is $66.03 \%$. 
Table 2. Achieved economic indicators of triticale production on 3 ha

\begin{tabular}{|c|c|c|c|c|c|c|}
\hline No. & Production year: 2012. & Amount & $\begin{array}{c}\text { Unit of } \\
\text { measure }\end{array}$ & Price & $\begin{array}{c}\text { Unit of } \\
\text { measure }\end{array}$ & $\begin{array}{c}\text { Amount of } \\
\text { EUR } \\
\text { for } 3 \text { ha }\end{array}$ \\
\hline $\mathbf{I}$ & Revenue & & & & & \\
\hline 1. & Triticale for 3 ha & 5.0 & $\mathrm{t} / \mathrm{ha}$ & 150.00 & $\mathrm{EUR} / \mathrm{t}$ & 2,250.00 EUR \\
\hline 2. & Straw from 3 ha & 5 & $\mathrm{t} / \mathrm{ha}$ & 19.50 & EUR/t & 292.50 EUR \\
\hline A) & Total revenue (1 to 2 ) for $3 \mathrm{ha}$ & & & & & 2,542.50 EUR \\
\hline 3. & Expenses & & & & & \\
\hline 4. & Seed for 3 ha & 750 & $\mathrm{~kg}$ & 0.20 & EUR/kg & 150 EUR \\
\hline 5. & Fertilizer & & & & & \\
\hline 6. & Manure for 3 ha & 15.0 & $\mathrm{t}$ & 4.00 & EUR/t & 60.00 EUR \\
\hline 7. & Urea & 600 & $\mathrm{~kg}$ & 0.30 & $\mathrm{EUR} / \mathrm{kg}$ & 180.00 EUR \\
\hline 8. & Foliar fertilization & 6 & $\mathrm{~kg}$ & 3.00 & EUR/kg & 18.00 EUR \\
\hline 9. & Pesticides & & & & & \\
\hline 10. & Meteor & 30 & $\mathrm{~g}$ & 0.15 & EUR/L & $4.50 \mathrm{EUR}$ \\
\hline 11. & Irrigation & & & & & \\
\hline 12. & Energy source for 3 ha & 15 & $\mathrm{~L}$ & 1.40 & EUR/L & 63.00 EUR \\
\hline 13. & Diesel fuel & 90 & $\mathrm{~L}$ & 1.40 & EUR/L & 126.00 EUR \\
\hline 14. & Maintenance of mechanization & 3 & ha & 19.00 & EUR/ha & 57.00 EUR \\
\hline 15. & Paid Services & & & & & \\
\hline 16. & Sowing & 3 & ha & 30.50 & EUR/ha & 91.50 EUR \\
\hline 17. & Harvest & 3 & ha & 52.00 & EUR/ha & 156.00 EUR \\
\hline 18. & Paid season work force & 50 & $\begin{array}{c}\text { working } \\
\text { hour }\end{array}$ & 1.50 & $\begin{array}{c}\text { EUR/ } \\
\text { working } \\
\text { hour }\end{array}$ & 75.00 EUR \\
\hline 19. & Other variable costs & & & & & \\
\hline B) & Total costs(3 to 19) & & & & & 981.00 EUR \\
\hline II & GAIN/LOSS & & & & & \\
\hline 20. & Total without incentive ( $A-B$ ) & & & & & 1,561.50 EUR \\
\hline 21. & Per ha without incentive $(20: 17)$ & & & & & 520.50 EUR \\
\hline 22. & Cost of grain in $\mathrm{kg}(20: 1)$ & & & & & 0.10 EUR \\
\hline 23. & Production efficiency (A : B) & & & & & 2.59 \\
\hline 24. & Revenue profitability (20:A) h 100 & & & & & $61.42 \%$ \\
\hline
\end{tabular}

Source: Authors'calculation based on data from Mičić, 2012

From Table 2 we can see that the average yield from triticale is $5.0 \mathrm{t} / \mathrm{ha}$ on the examined farm $\mathrm{A}$ and that it ranged from $4.5 \mathrm{t} / \mathrm{ha}$ to $5.5 \mathrm{t} / \mathrm{ha}$. Total realized gain on 3 ha is $1,561.50 \mathrm{EUR}$, profitability of revenue is $61.42 \%$. 
Table 3. Achieved economic indicators of wheat production on 2 ha

\begin{tabular}{|c|c|c|c|c|c|c|}
\hline No. & Production year: 2012 & Amount & $\begin{array}{c}\text { Unit of } \\
\text { measure }\end{array}$ & Price & $\begin{array}{c}\text { Unit of } \\
\text { measure }\end{array}$ & $\begin{array}{c}\text { Amount of } \\
\text { EUR for } 2 \text { ha }\end{array}$ \\
\hline I & Revenue & & & & & \\
\hline 1. & Wheat from 2 ha & 5 & $\mathrm{t} / \mathrm{ha}$ & 170.00 & $\mathrm{EUR} / \mathrm{t}$ & 1,700.00 EUR \\
\hline 2. & Straw from 2 ha & 5 & $\mathrm{t} / \mathrm{ha}$ & 16.90 & $\mathrm{EUR} / \mathrm{t}$ & 169.00 EUR \\
\hline A) & Total revenue(1 to 2)for 2 ha & & & & & 1,869.00 EUR \\
\hline 3 & Expenses & & & & & \\
\hline 4. & Seed for 2 ha & 500 & $\mathrm{~kg}$ & 0.20 & EUR/kg & 100.00 EUR \\
\hline 5. & Fertilizer & & & & & \\
\hline 6. & Manure for $2 \mathrm{ha}$ & 10 & $\mathrm{t}$ & 4.00 & $\mathrm{EUR} / \mathrm{t}$ & 40.00 EUR \\
\hline 7. & Urea & 400 & $\mathrm{~kg}$ & 0.30 & EUR/kg & $120.00 \mathrm{EUR}$ \\
\hline 8. & Foliar fertilization & 4 & $\mathrm{~kg}$ & 3.00 & EUR/kg & $12.00 \mathrm{EUR}$ \\
\hline 9. & Pesticides & & & & & \\
\hline 10. & Meteor & 20 & $\mathrm{~g}$ & 0.15 & EUR/L & 3.00 EUR \\
\hline 11. & Irrigation & & & & & \\
\hline 12. & Energy source for 2 ha & 15 & $\mathrm{~L}$ & 1.40 & EUR/L & 42.00 EUR \\
\hline 13. & Diesel fuel & 60 & $\mathrm{~L}$ & 1.40 & EUR/L & 84.00 EUR \\
\hline 14. & Maintenance of mechanization & 2 & ha & 19.00 & EUR/ha & 38.00 EUR \\
\hline 15. & Paid services & & ha & & & \\
\hline 16. & Plowing & 2 & ha & & EUR/ha & 0 EUR \\
\hline 17. & Preparing the ground & 2 & ha & & EUR/ha & 0 EUR \\
\hline 18. & Sowing & 2 & ha & 30.00 & EUR/ha & $60.00 \mathrm{EUR}$ \\
\hline 19. & Harvest & 2 & ha & 55.00 & EUR/ha & 110.00 EUR \\
\hline 20. & Paid season work force & 30 & $\begin{array}{c}\text { working } \\
\text { hours }\end{array}$ & 1.50 & $\begin{array}{c}\text { EUR/ } \\
\text { working } \\
\text { hour }\end{array}$ & 45.00 EUR \\
\hline 21. & Other variable costs & & & & & \\
\hline B) & Total costs(3 to 21) & & & & & 654.00 EUR \\
\hline II & GAIN/LOSS & & & & & \\
\hline 22. & Total without incentive $(A-B)$ & & & & & 1,215.00 EUR \\
\hline 23. & Per ha without incentive $(22: 17)$ & & & & & 607.50 EUR \\
\hline 24. & Cost of grain kg (22: 1) & & & & & 0.12 EUR \\
\hline 25. & Production efficiency (A : B) & & & & & 2.86 \\
\hline 26. & Revenue profitability (22: A) h 100 & & & & & $65.00 \%$ \\
\hline
\end{tabular}

Source: Authors'calculation based on data from Mičić, 2012

From Table 3 we can see that the average yield of wheat is $5.0 \mathrm{t} / \mathrm{ha}$ on the examined farm and that it ranged from 4.5 t/ha to $5.5 \mathrm{t} / \mathrm{ha}$. Total profit on 2 ha is 1,215.00 EUR and revenue profitability is $65.00 \%$. 
Table 4. Achieved economic indicators of barley production on 3 ha

\begin{tabular}{|c|c|c|c|c|c|c|}
\hline No. & Production year: 2012 & Amount & $\begin{array}{c}\text { Unit of } \\
\text { measure }\end{array}$ & Price & $\begin{array}{l}\text { Unit of } \\
\text { Measure }\end{array}$ & $\begin{array}{c}\text { Amount of } \\
\text { EUR } \\
\text { for } 3 \text { ha } \\
\end{array}$ \\
\hline $\mathbf{I}$ & Revenue & & & & & \\
\hline 1. & Barley from 3 ha & 4 & $\mathrm{t} / \mathrm{ha}$ & 170.00 & $\mathrm{EUR} / \mathrm{t}$ & $\begin{array}{c}2,040.00 \\
\text { EUR }\end{array}$ \\
\hline 2. & Straw from 3 ha & 5 & $\mathrm{t} / \mathrm{ha}$ & 17.04 & EUR/t & 255.60 EUR \\
\hline A) & Total revenue( 1 to 2 ) for 3 ha & & & & & $\begin{array}{c}\mathbf{2 , 2 9 5 . 6 0} \\
\text { EUR }\end{array}$ \\
\hline 3. & Expenses & & & & & \\
\hline 4. & Seed for 3 ha & 750 & $\mathrm{~kg}$ & 0.20 & $\mathrm{EUR} / \mathrm{kg}$ & $150.00 \mathrm{EUR}$ \\
\hline 5. & Fertilizer & & & & & \\
\hline 6. & Manure for $3 \mathrm{ha}$ & 15.0 & $\mathrm{t}$ & 4.00 & $\mathrm{EUR} / \mathrm{t}$ & 60.00 EUR \\
\hline 7. & Urea & 600 & $\mathrm{~kg}$ & 0.30 & EUR/kg & 180.00 EUR \\
\hline 8. & Foliar fertilization & 6 & $\mathrm{~kg}$ & 3.00 & $\mathrm{EUR} / \mathrm{kg}$ & $18.00 \mathrm{EUR}$ \\
\hline 9. & Pesticides & & & & & \\
\hline 10. & Meteor & 30 & $\mathrm{~g}$ & 0.15 & EUR/L & 4.50 EUR \\
\hline 11. & Irrigation & & & & & \\
\hline 12. & Energy source for $3 \mathrm{ha}$ & 15 & $\mathrm{~L}$ & 1.40 & EUR/L & $63.00 \mathrm{EUR}$ \\
\hline 13. & Diesel fuel & 90 & $\mathrm{~L}$ & 1.40 & EUR/L & 126.00 EUR \\
\hline 14. & Maintenance of mechanization & 3 & ha & 19.00 & EUR/ha & 57.00 EUR \\
\hline 15. & Paid services & & & & & \\
\hline 16. & Plowing & & ha & & EUR/ha & 0 EUR \\
\hline 17. & Preparation of ground & 3 & ha & & EUR/ha & 0 EUR \\
\hline 18. & Sowing & 3 & ha & 30.50 & EUR/ha & $91.50 \mathrm{EUR}$ \\
\hline 19. & Harvest & 3 & ha & 55.00 & EUR/ha & 165.00 EUR \\
\hline 20. & Paid season work force & 44 & r. hours & 1.50 & EUR/r.s. & 66.00 EUR \\
\hline 21. & Other variable costs & & & & & \\
\hline B) & Total costs(3 to 21) & & & & & 981.00 EUR \\
\hline II & GAIN/LOSS & & & & & \\
\hline 22. & Total without incentive ( $A-B$ ) & & & & & $\begin{array}{c}\mathbf{1 , 3 1 4 . 6 0} \\
\text { EUR } \\
\end{array}$ \\
\hline 23. & Per ha without incentive $(22: 17)$ & & & & & 438.20 EUR \\
\hline 24. & Cost of grain kg (22: 1) & & & & & 0.11 EUR \\
\hline 25. & Production efficiency (A : B) & & & & & 2.34 \\
\hline 26. & Revenue profitability (22: A) h 100 & & & & & $57.26 \%$ \\
\hline
\end{tabular}

Source: Authors' calculation based on data from Mičić, 2012

From Table 4 we can see that the average yield of barley on the farm was $4.0 \mathrm{t} / \mathrm{ha}$ and that it ranged from $3.5 \mathrm{t} / \mathrm{ha}$ to $4.5 \mathrm{t} / \mathrm{ha}$. Total gain on 3 ha is 1,314.60 EUR, production efficiency is 2.34 and revenue profitability is $57.26 \%$. It can also be seen that revenue from grain on farm $\mathrm{A}$ is $50 \mathrm{t}$, it moved from $3.5 \mathrm{t} /$ ha to $7.5 \mathrm{t} / \mathrm{ha}$ and from total amounts of plant production farm A used 1/3 to feed the heard while 2/3 was sold on the market. 


\section{Pricelist of mixture for feeding pigs on farm $\mathrm{A}$}

During price calculation of the mixture from own produced grain for breeding pigs on farm, price of all products was taken into consideration, calculated in tons $(\mathrm{t})$. More data about it is shown in Table 5.

Table 5. Prices of concentrate mixtures on farm A

\begin{tabular}{|l|c|}
\hline $\begin{array}{c}\text { PRICELIST OF FEED MIXTURE FOR PIGS ON A FARM IN } \\
\text { KOSOVO AND METOHIJA IN GRAČANICA IN 2012 }\end{array}$ & Price EUR/kg \\
\hline Pre-starter mixture for feeding pigs up to 10kg (PS) & 0.27 \\
\hline Grover mixture for feeding pigs from 15 to $25 \mathrm{~kg}$ (SS) & 0.22 \\
\hline Starter mixture for feeding pigs up to $15 \mathrm{~kg}$ (SG) & 0.24 \\
\hline Mixture for feeding fattened pigs from 25 to $60 \mathrm{~kg}$ (TS-1) & 0.20 \\
\hline Mixture for feeding fattened pigs from 60 to $100 \mathrm{~kg}$ (TS-2) & 0.19 \\
\hline Mixture for feeding pregnant gilts and sows (SK) & 0.17 \\
\hline Mixture feeding lactating sows and boars (SKD) & 0.16 \\
\hline
\end{tabular}

Source: Authors' calculation based on data from Mičić, 2012

From Table 5 we can see that the feeding mixture for all categories of pigs farm A produces by itself in powder form in their blenders at producers prices.

Price of the concentrate mixture on the farm was calculated by average exchange rate of the National Bank of Serbia in EUR/kg/116 RSD in 2012.

\section{Productivity of sows and raising piglets on farm $A$}

Farm had ten sows of landrace breed which had two farrowing a year with an average of ten piglets per brood, i.e. 20 piglets per sow a year and one boar of the Yorkshire breed.

Piglets were weaned after 21 days with an average body weight of $5.24 \mathrm{~kg}$. Their breeding lasted 38 days after that until they reached $25 \mathrm{~kg}$, with the achieved daily growth of $0.52 \mathrm{~kg} /$ day (Table 6).

Table 6. Productivity of sows and raising piglets up to $25 \mathrm{~kg}$ in 2012 on farm A

Livestock number on farm 10

Racial composition Landrace + Yorkshire

Time for fattening in years (two rounds)

Average 20 piglets/sow a year

Age of piglets when weaning 21 day

\section{Lowland region}

Input weight

Exiting weight $25 \mathrm{~kg}$

Weight of a piglet after weaning $5.24 \mathrm{~kg}$

Raising piglets 38 days x $0.52 \mathrm{~kg}$ /day 


\begin{tabular}{|c|c|c|c|c|c|c|}
\hline \multirow[b]{2}{*}{$\mathbf{I}$} & \multirow[b]{2}{*}{ Revenue } & \multicolumn{3}{|c|}{ Amount } & \multirow{2}{*}{$\begin{array}{c}\text { Price per } \\
\text { unit } \\
\text { EUR } \\
\end{array}$} & \multirow{2}{*}{$\begin{array}{l}\text { To t a l } \\
\text { EUR }\end{array}$} \\
\hline & & $\begin{array}{l}\text { Number } \\
\text { of pigs }\end{array}$ & $\begin{array}{c}\mathrm{kg} / \\
\text { livestock }\end{array}$ & $\begin{array}{c}\text { Total } \\
\text { kg }\end{array}$ & & \\
\hline 1 & Raising piglets & 200 & 25 & 5.000 & 2.80 & $14,000.00$ \\
\hline 2 & Incentive RS & 200 & - & - & - & - \\
\hline 3 & Incentive RS (sow) & 10 & - & - & - & - \\
\hline 4 & Insurance reimbursement & & & & & - \\
\hline A) & Tot a l( 1 to 4$)$ & & & & & $14,000.00$ \\
\hline II & EXPENSES & & & & & \\
\hline 5 & Feeding piglets & & & & & \\
\hline 6 & $\begin{array}{l}\text {-pre-starter }(0.2 \mathrm{~kg} / \text { day } \mathrm{x} 10 \text { days } \mathrm{x} 200 \\
\text { livestock })\end{array}$ & 200 & 2 & 400 & 0.2695 & 107.80 \\
\hline 7 & $\begin{array}{l}\text {-SS (to } 15 \mathrm{~kg})(0.5 \mathrm{~kg} / \text { day x } 13 \text { days x } 200 \\
\text { livestock) }\end{array}$ & 200 & 6.5 & 1.300 & 0.2415 & 313.95 \\
\hline 8 & $\begin{array}{l}\text {-SG }(15-25 \mathrm{~kg})(1.56 \mathrm{~kg} / \text { day } \mathrm{x} 15 \text { days } \\
\text { x200 livestock) }\end{array}$ & 200 & 23.4 & 4.680 & 0.2190 & $1,024.92$ \\
\hline 9 & $\begin{array}{l}\text { Feeding sows }(2.5 \mathrm{~kg} / \text { day x365days x } 10 \\
\text { livestock) }\end{array}$ & 10 & 912.5 & 9.125 & 0.1666 & $1,520.23$ \\
\hline 10 & $\begin{array}{l}\text { Feeding boars }(2.2 \mathrm{~kg} / \text { day x } 365 \text { days } \mathrm{x} \\
\text { 1livestock })\end{array}$ & 1 & 803 & 803 & 0.1616 & 129.76 \\
\hline B) & Total feed( 5 to 10$)$ & & & & & $3,096.66$ \\
\hline 11 & Losses in feeding $1 \%$ & & 50 & & 2.40 & 120.00 \\
\hline 12 & Water and medicine - sow & 10 & - & - & 10.00 & 100.00 \\
\hline 13 & Human labor (personal or someone else's) & $\begin{array}{c}\text { working } \\
\text { day }\end{array}$ & & 40 & 10.00 & 400.00 \\
\hline 14 & $\begin{array}{l}\text { Amortization of the heard (400- } \\
100=300 \times 20 \%)\end{array}$ & 10 & - & - & 60.00 & 600.00 \\
\hline 15 & Amortization of the facility and equipment & & & 8.000 & $2.5 \%$ & 200.00 \\
\hline 16 & Total direct costs ( 5 to 15$)$ & & & & & $4,906.66$ \\
\hline 17 & Indirect costs of the farm & & & & & 490.00 \\
\hline C) & Total costs( 5 to 17) & & & & & $5,006.66$ \\
\hline III & GAIN/LOSS & & & & & \\
\hline 18 & On a farm without $r$ incentive $(A-C)$ & & & & & $8,993.34$ \\
\hline 19 & Per pig without incentive $(18: 3)$ & & & & & 899.33 \\
\hline 20 & Price of a $\mathrm{kg}(\mathrm{C}: \mathbf{1})$ & & & & & 1.00 \\
\hline 21 & Production efficiency (A : C) & & & & & 2.79 \\
\hline 22 & Revenue profitability (18: A) h 100 & & & & & $64.24 \%$ \\
\hline
\end{tabular}

Source: Authors' calculation based on data from Mičić, 2012

From Table 6 we can see that the average weight of piglets on the farm in Gračanica is $25 \mathrm{~kg} /$ livestock and that it ranged from 24 to $26 \mathrm{~kg}$ / livestock, with the achieved average price of $1 € / \mathrm{kg}$ and the value of 1 pig was $25.00 €$. Total achieved gain for 200 
piglets was 8,993.34 $€$, production efficiency 2.79 and revenue profitability $64.24 \%$. In Table 7 there are 40 fattened livestock in 2012 on a family farm A.

\section{Fattened pigs on farm $A$}

Farm A fattens 40 pigs a year in 4 turns and sells them ex-loaded on the farm as well as excess piglets. Farm A breeds around 200 piglets a year of average weight of $25 \mathrm{kf}$ and raises gilts for themselves. Farm A sells $80 \%$ of piglets free loaded after they have achieved $25 \mathrm{~kg}$ of mass at the price of 2.8 euros, in 2012 (Table 7), as well as total achieved economic indicators (Table 8).

Table 7. Achieved economic indicators in fattened pigs on farm A for 2012

\begin{tabular}{|c|c|c|c|c|c|c|c|}
\hline No. & $\begin{array}{l}\text { Production year: } \\
2012 .\end{array}$ & Amount & \multicolumn{3}{|c|}{ Stopa konverzije kg hrane: } & 2.68 & kg growth \\
\hline 1. & $\begin{array}{l}\text { Fattening period: } \\
\text { Jan-Dec }\end{array}$ & - & $\begin{array}{l}\text { Unit of } \\
\text { Measure }\end{array}$ & \multicolumn{2}{|c|}{ Mortality rate in feeding: } & $2.43 \%$ & \\
\hline 2. & $\begin{array}{l}\text { Number of pigs } \\
\text { placed in fattening: }\end{array}$ & 41 & livestock & & & & \\
\hline 3. & $\begin{array}{l}\text { Average weight of } \\
\text { fattened: }\end{array}$ & 100 & $\begin{array}{c}\mathrm{kg} / \\
\text { livestock }\end{array}$ & & & & \\
\hline 4. & Fattening time: & 87 & days & & & & \\
\hline $\mathbf{I}$ & Revenue & - & - & Price & $\begin{array}{c}\text { Unit of } \\
\text { Measure }\end{array}$ & Amount/€ & $\begin{array}{l}\text { Amount }(€ / \\
\text { livestock) }\end{array}$ \\
\hline 5. & $\begin{array}{l}\text { Fattened pigs ( } \\
3 \times 5)\end{array}$ & 40.00 & livestock & 1.58 & $\mathrm{EUR} / \mathrm{kg}$ & 6.320 .00 & 158.00 EUR \\
\hline 6. & Manure & 20.00 & $\mathrm{t}$ & 4.00 & $\mathrm{EUR} / \mathrm{t}$ & 80.00 & 2.00 EUR \\
\hline 7. & $\begin{array}{l}\text { Subventions per } \\
\text { pig }\end{array}$ & 40.00 & livestock & 8.70 & $\begin{array}{c}\text { EUR/ } \\
\text { livestock }\end{array}$ & 348.00 & 8.70 EUR \\
\hline A) & $\begin{array}{l}\text { Total revenue ( } 1 \\
\text { to } 7)\end{array}$ & - & & & & 6.748 .00 & 168.70 EUR \\
\hline II & Expenses & - & $\begin{array}{l}\text { Unit of } \\
\text { measure }\end{array}$ & Price & $\begin{array}{l}\text { Unit of } \\
\text { measure }\end{array}$ & Amount $/ €$ & $\begin{array}{c}\text { (EUR/ } \\
\text { livestock) }\end{array}$ \\
\hline 8. & $\begin{array}{l}\text { Piglets (average } \\
\text { livestock) }\end{array}$ & 25.00 & $\begin{array}{c}\mathrm{kg} / \\
\text { livestock }\end{array}$ & & & & \\
\hline 9. & Piglets $(2 \times 8)$ & $1,025.00$ & $\begin{array}{c}\mathrm{kg} / \\
\text { livestock }\end{array}$ & 1.00 & $\mathrm{EUR} / \mathrm{kg}$ & $1,025.00$ & 25.00 EUR \\
\hline 10. & \multicolumn{2}{|c|}{ Own mixture of concentrate } & & & & & \\
\hline 11. & \multicolumn{2}{|c|}{$\begin{array}{l}\text { TS1 (from } 25-60 \mathrm{~kg})(2.15 \mathrm{~kg} / \text { day } \\
\mathrm{x} 41 \text { days } \mathrm{x} 40 \text { g.) } 3.526\end{array}$} & & 0.20 & $\mathrm{EUR} / \mathrm{kg}$ & 705.00 & 17.63 EUR \\
\hline 12. & \multicolumn{2}{|c|}{$\begin{array}{l}\text { TS2 (from } 60-100 \mathrm{~kg})(2.45 \mathrm{~kg} / \\
\text { day x46days x40 g.) } 4.508\end{array}$} & & 0.19 & EUR/kg & 856.50 & 21.41 EUR \\
\hline 13. & $\begin{array}{l}\text { Average daily per } \\
\text { pig }\end{array}$ & 2.31 & $\mathrm{~kg} / \mathrm{EUR}$ & & & & \\
\hline 14. & $\begin{array}{l}\text { Mechanical } \\
\text { work }(7 \mathrm{~h} 14)\end{array}$ & & $\mathrm{kg} / \mathrm{EUR}$ & 2.00 & EUR/kg & 80.00 & 2.00 EUR \\
\hline 15. & $\begin{array}{l}\text { Water per } \\
\operatorname{pig}(\mathbf{1 5 x} 4) \times \\
\mathbf{8 : 1 . 0 0 0}\end{array}$ & 10 & L/day & 1.25 & $\mathrm{EUR} / \mathrm{m}^{3}$ & 43.50 & $1.08 \mathrm{EUR}$ \\
\hline 16. & \multicolumn{2}{|c|}{$\begin{array}{l}\text { Veterinary services and } \\
\text { medicine }(7 \times 16)\end{array}$} & & 1.00 & $\begin{array}{c}\text { EUR/ } \\
\text { livestock }\end{array}$ & 40.00 & 1.00 EUR \\
\hline
\end{tabular}




\begin{tabular}{|c|c|c|c|c|c|c|}
\hline \multirow{2}{*}{$\begin{array}{l}\text { No. } \\
17 .\end{array}$} & \begin{tabular}{|c|} 
Production year: \\
2012.
\end{tabular} & \multirow[t]{2}{*}{ Amount } & \multicolumn{2}{|c|}{ Stopa konverzije kg hrane: } & \multirow{2}{*}{$\begin{array}{c}\mathbf{2 . 6 8} \\
600.00\end{array}$} & \multirow{2}{*}{$\begin{array}{c}\text { kg growth } \\
15.00 \text { EUR }\end{array}$} \\
\hline & Human labor(3 x 5) x 17 & & 0.15 & $\begin{array}{c}\text { EUR/ } \\
\text { livestock }\end{array}$ & & \\
\hline 18. & \multicolumn{2}{|l|}{ Indirect costs( 7 x 18) } & 1.40 & $\begin{array}{c}\text { EUR/ } \\
\text { livestock }\end{array}$ & 56.00 & $1.40 \mathrm{EUR}$ \\
\hline 19. & \multicolumn{2}{|c|}{$\begin{array}{l}\text { Amortization of facilities and } \\
\text { equipment }(7 \times 19)\end{array}$} & 2.00 & $\begin{array}{c}\text { EUR/ } \\
\text { livestock }\end{array}$ & 80.00 & 2.00 EUR \\
\hline B) & \multicolumn{2}{|l|}{ Total costs $(9$ to 30$)$} & & & $3,486.00$ & 86.52EUR \\
\hline III & \multicolumn{2}{|l|}{ GAIN/LOSS } & & & & \\
\hline 20. & \multicolumn{2}{|c|}{ On farm with incentive(A - B) } & & & 3.262 .00 & 81.55EUR \\
\hline 21. & \multicolumn{2}{|l|}{ Price in $\mathrm{kg} \mathrm{B}:(3 \times 5)$} & & & 0.87 & \\
\hline 22. & \multicolumn{2}{|c|}{ Production efficiency (A : B) } & & & 1.94 & \\
\hline 23. & \multicolumn{2}{|c|}{$\begin{array}{l}\text { Revenue profitability }(20: A) \mathrm{x} \\
100\end{array}$} & & & $48.34 \%$ & \\
\hline
\end{tabular}

Source: Authors' calculation based on data from Mičić, 2012

From Table 7 we can see that the value of one fattened pig was $87.00 \mathrm{EUR} / \mathrm{pig}$. Total achieved gain for 40 fattened pigs is 3,262.00 EUR, production efficiency 1.94 and revenue profitability $48.34 \%$.

Table 8. Total achieved economic results in agricultural and livestock production on farm A

\begin{tabular}{|c|l|c|c|c|c|c|c|c|}
\hline No. & \multicolumn{2}{|l|}{$\begin{array}{l}\text { Farm in Gračanica } \\
\text { Production year: }\end{array}$} & Revenue & Amount & $\begin{array}{c}\text { Unit of } \\
\text { measure }\end{array}$ & Price & $\begin{array}{c}\text { Unit of } \\
\text { measure }\end{array}$ & $\begin{array}{c}\text { Amount in EUR } \\
\text { for 2 ha }\end{array}$ \\
\hline I & REVENUES & For & & & e & & & \\
\hline $\mathbf{1 .}$ & $\begin{array}{l}\text { Corn from2 } \\
\text { ha }\end{array}$ & 2 & 6.5 & 7.1844 & $\mathrm{t} / \mathrm{ha}$ & 130.00 & EUR/t & $933.97 \mathrm{EUR}$ \\
\hline $\mathbf{2 .}$ & $\begin{array}{l}\text { Cornstalks } \\
\text { from2ha }\end{array}$ & 2 & 9 & 18 & $\mathrm{t} / \mathrm{ha}$ & 17.98 & $\mathrm{EUR} / \mathrm{t}$ & $323.70 \mathrm{EUR}$ \\
\hline $\mathbf{3 .}$ & $\begin{array}{l}\text { Triticale from } \\
\text { h ha }\end{array}$ & 3 & 5 & 11.618 & $\mathrm{t} / \mathrm{ha}$ & 150.00 & $\mathrm{EUR} / \mathrm{t}$ & $1,742.70 \mathrm{EUR}$ \\
\hline $\mathbf{4 .}$ & $\begin{array}{l}\text { Straw from } \\
\text { 3 ha }\end{array}$ & 3 & 5 & 15 & $\mathrm{t} / \mathrm{ha}$ & 19.50 & $\mathrm{EUR} / \mathrm{t}$ & $292.50 \mathrm{EUR}$ \\
\hline $\mathbf{5 .}$ & $\begin{array}{l}\text { Wheat from } \\
\text { 2 ha }\end{array}$ & 2 & 5 & 8.6036 & $\mathrm{t} / \mathrm{ha}$ & 170.00 & $\mathrm{EUR} / \mathrm{t}$ & $1,462.61 \mathrm{EUR}$ \\
\hline $\mathbf{6 .}$ & $\begin{array}{l}\text { Straw from } \\
\text { 2 ha }\end{array}$ & 2 & 5 & 10 & $\mathrm{t} / \mathrm{ha}$ & 16.90 & $\mathrm{EUR} / \mathrm{t}$ & $169.00 \mathrm{EUR}$ \\
\hline $\mathbf{7 .}$ & $\begin{array}{l}\text { Barleyfrom3 } \\
\text { ha }\end{array}$ & 3 & 4 & 6.141 & $\mathrm{t} / \mathrm{ha}$ & 170.00 & $\mathrm{EUR} / \mathrm{t}$ & $1,043.97 \mathrm{EUR}$ \\
\hline $\mathbf{8 .}$ & $\begin{array}{l}\text { Strawfrom3 } \\
\text { ha }\end{array}$ & 3 & 5 & 15 & $\mathrm{t} / \mathrm{ha}$ & 17.04 & $\mathrm{EUR} / \mathrm{t}$ & $255.60 \mathrm{EUR}$ \\
\hline $\mathbf{9 .}$ & $\begin{array}{l}\text { Piglets } \\
\text { livestock }\end{array}$ & 159 & 25 & 3.975 & $\mathrm{~kg}$ & 2.80 & $\mathrm{EUR} / \mathrm{kg}$ & $11,130.00 \mathrm{EUR}$ \\
\hline $\mathbf{1 0 .}$ & Fattened pigs & 40 & 100 & 4.000 & $\mathrm{~kg}$ & 1.58 & $\mathrm{EUR} / \mathrm{kg}$ & $6,320.00 \mathrm{EUR}$ \\
\hline
\end{tabular}




\begin{tabular}{|c|c|c|c|c|c|c|c|c|}
\hline 11. & $\begin{array}{l}\text { Manure per } \\
\text { livestock }\end{array}$ & 0.5 & 40 & 20 & $\mathrm{t}$ & 4.00 & $\mathrm{EUR} / \mathrm{t}$ & 80.00 EUR \\
\hline 12. & \multicolumn{2}{|c|}{ Subventions livestock } & & 40 & livestock & 8.70 & $\begin{array}{c}\text { EUR/ } \\
\text { livestock }\end{array}$ & 348.00 EUR \\
\hline A) & \multicolumn{2}{|c|}{$\begin{array}{l}\text { Total revenue (1 to } \\
\text { 12) }\end{array}$} & & & & & & 24,102.05 EUR \\
\hline II & \multicolumn{2}{|c|}{ Expenses } & & & & & & \\
\hline 13. & \multicolumn{2}{|l|}{ Cornfrom 2 ha } & & & & & & 684.00 EUR \\
\hline 14. & \multicolumn{2}{|c|}{ Triticale for 3 ha } & & & & & & 981.00 EUR \\
\hline 15. & \multicolumn{2}{|c|}{ Wheat for 2 ha } & & & & & & 654.00 EUR \\
\hline 16. & \multicolumn{2}{|c|}{ Barley for 3 ha } & & & & & & 981.00 EUR \\
\hline 17. & \multicolumn{2}{|c|}{ Upbringing piglets } & & & & & & 3,300.72 EUR \\
\hline 18. & \multicolumn{2}{|c|}{$\begin{array}{l}\text { Fattening pigs } 40 \\
\text { livestock }\end{array}$} & & & & & & 900.00 EUR \\
\hline В) & \multicolumn{3}{|c|}{ Total expenses(13 to 18$)$} & & & & & 7,500.72 EUR \\
\hline II & \multicolumn{3}{|l|}{ GAIN/LOSS } & & & & & \\
\hline 19. & \multicolumn{3}{|c|}{ Total with incentive ( A - B ) } & & & & & 16,601.33 EUR \\
\hline 20. & \multicolumn{3}{|c|}{ Production efficiency (A:B) } & & & & & 3.21 \\
\hline 21. & \multicolumn{4}{|c|}{ Revenue profitability $(19:$ A)h100 } & & & & $68.88 \%$ \\
\hline
\end{tabular}

Source: Authors'calculation based on data from Mičić, 2012

From Table 8 we can see that in 2012 the farm achieved total revenue of 24,102.05 EUR; expenses in the amount of 7,500.72 EUR; gain in the amount of 16,601.33 EUR; in agriculture and livestock production and sale production efficiency 3.21 ; a d revenue profitability $68.88 \%$.

Pricelist of concentrate mixtures on farm B for feeding pigs of all categories is shown in Table 9.

Table 9.Price of concentrate mixture on farm B

\begin{tabular}{|l|c|}
\hline $\begin{array}{c}\text { PRICELEST OF MIXTURE FOR FEEDING PIGS ON A FARM ON } \\
\text { KOSOVO AND METOHIJA IN GRAČANICA IN 2012 }\end{array}$ & PRICE EUR/kg \\
\hline Pre-starter mixture for feeding piglets to $10 \mathrm{~kg}$ (PS) & 0.48 \\
\hline Grover mixture for feeding piglets from 15 to $25 \mathrm{~kg}$ (SS) & 0.34 \\
\hline Starter mixture for feeding piglets to $15 \mathrm{~kg}$ (SG) & 0.33 \\
\hline Mixture for feeding fattened pigs from 25 to $60 \mathrm{~kg}$ (TS-1) & 0.28 \\
\hline Mixture for feeding fattened pigs from 60 to $100 \mathrm{~kg}$ (TS-2) & 0.26 \\
\hline Mixture for feeding pregnant gilts and sows (SK) & 0.25 \\
\hline Mixture for feeding lactating sows and boars (SKD) & 0.29 \\
\hline
\end{tabular}

Source: Authors'calculation based on data from Mičić, 2012

The Farm has its own blenders that operates independently and is located by the entrance gate and by the above mentioned pricelist of mixture entrusts farm B. 


\section{Productivity of sows and upbringing of piglets on farm B}

Farm B has 1,500 sows Landrace + Yorkshire which had two farrowing a year in the average of 10.3 raised piglets per breed, i.e. 20.6 piglets a year. Piglets are weaning after 28 days with the average body weight of $6.6 \mathrm{~kg}$. Their upbringing lasted 34 days after that up to body weight of $25 \mathrm{~kg}$, with the achieved daily growth of $0.54 \mathrm{~kg}$ a day.

More data on productivity of sows and raising piglets on farm B is given in Table 10 .

Table 10. Productivity of sows and raising piglets up to $25 \mathrm{~kg}$ on farm B in 2012

Livestock Number on farm 1,500

Racial composition Landrace +Yorkshire

Fattening weight in years (two cycles)

Average 20.6 piglets/pig a year

Age of piglets after weaning 28 days

\section{Lowland region}

Entrance weight

Exiting weight $25 \mathrm{~kg}$

Weight of a piglet after weaning $6.6 \mathrm{~kg}$

Raising piglets 34 days x $0.54 \mathrm{~kg} /$ day

\begin{tabular}{|c|c|c|c|c|c|c|}
\hline I & REVENUE & $\begin{array}{l}\text { Number of } \\
\text { livestock }\end{array}$ & $\mathrm{kg} /$ livestock & Total kg & Price unit & Total $\boldsymbol{\epsilon}$ \\
\hline 1 & $\begin{array}{l}\text { Raising piglets put for } \\
\text { fattening: }\end{array}$ & 30,600 & & & & \\
\hline 2 & $\begin{array}{l}\text { Average end weight of } \\
\text { piglets: }\end{array}$ & 30,000 & 25 & 750,000 & 2.39 & $1,792,500.00$ \\
\hline 3 & Manure (sows)total & 1,500 & 500 & 750,000 & 0.01 & $7,500.00$ \\
\hline $\mathbf{A}$ & Tot a l (1 to 3) & & & & & $1,800,000.00$ \\
\hline II & EXPENSES & & & & & \\
\hline 5 & $\begin{array}{l}\text { Feeding piglets/ mixture } \\
\text { according to pricelist Table } \\
8 \text {. }\end{array}$ & & & & & \\
\hline 6 & $\begin{array}{l}\text {-pre-starter }(0.2 \mathrm{~kg} / \text { day } \\
\text { x10days x30,000 pigs })\end{array}$ & 30,000 & 2 & 60,000 & 0.48 & $28,800.00$ \\
\hline 7 & $\begin{array}{l}\text {-SP1 (to } 15 \mathrm{~kg})(0.6 \mathrm{~kg} / \text { day } \\
\mathrm{x} 11 \text { days x } 30,000 \text { pigs })\end{array}$ & 30,000 & 6.6 & 198,000 & 0.34 & $67,320.00$ \\
\hline 8 & $\begin{array}{l}\text {-SP2 }(15-25 \mathrm{~kg})(1.8 \mathrm{~kg} / \text { day } \\
\text { x13days x30,000 pigs })\end{array}$ & 30,000 & 23.4 & 702,000 & 0.32 & $224,640.00$ \\
\hline 9 & $\begin{array}{l}\text { Feed to sow }(4.5 \mathrm{~kg} / \text { day } \\
\text { x } 46 \text { days } x 1,500 \text { pigs })\end{array}$ & 1,500 & 207 & 310,500 & 0.28 & $86,940.00$ \\
\hline 10 & $\begin{array}{l}\text { Feeding a sow }(4.5 \mathrm{~kg} / \text { day } \\
\text { x365days x } 1,500 \text { pigs })\end{array}$ & 1,500 & $1,642.5$ & $2,463,750$ & 0.26 & $640,575.00$ \\
\hline 11 & $\begin{array}{l}\text { Feeding a boar }(4 \mathrm{~kg} / \text { day } \\
\text { x365days x } 25 \text { pigs })\end{array}$ & 25 & 1,460 & 36,500 & 0.26 & $9,490.00$ \\
\hline В) & Total feed (5 to 11) & & $3,341.5$ & $3,770,750$ & & $1,057,765.00$ \\
\hline 12 & Loss in fattening piglets $2 \%$ & & - & - & & $36,000.00$ \\
\hline 13 & Under vacuum & 30,000 & & & 1.00 & $30,000.00$ \\
\hline 14 & $\begin{array}{l}\text { Water and medicine - sow } \\
\text { and boars }\end{array}$ & 1,525 & - & - & 20.00 & $30,500.00$ \\
\hline
\end{tabular}




\begin{tabular}{|c|l|c|c|c|c|c|}
\hline 15 & $\begin{array}{l}\text { Human labor (personal } \\
\text { someone else's) }\end{array}$ & $\begin{array}{c}\text { working } \\
\text { day }\end{array}$ & & 365 & 400.00 & $146,000.00$ \\
\hline 16 & $\begin{array}{l}\text { Amortization of pigs (450 } \\
-150=300 x 20 \%)\end{array}$ & 1,525 & - & - & 60.00 & $91,500.00$ \\
\hline 17 & $\begin{array}{l}\text { Amortization of facilities } \\
\text { and equipment }\end{array}$ & & & $1,449,275$ & $3 \%$ & $43,478.00$ \\
\hline 18 & Total direct costs(5 to 18) & & & & & $1,494,718.00$ \\
\hline 19 & Indirect costs of the farm & & & & & $93,559.00$ \\
\hline C) & Total costs $(18+19)$ & & & & & $\mathbf{1 , 5 2 8 , 8 0 2 . 0 0}$ \\
\hline III & GAIN/LOSS & & & & & $271,198.00$ \\
\hline 20 & $\begin{array}{l}\text { On a farm without } \\
\text { incentive(A-C) }\end{array}$ & & & & & 180.79 \\
\hline 21 & $\begin{array}{l}\text { Per pig without incentive } \\
(20: 3)\end{array}$ & & & & & 2.04 \\
\hline 22 & Price for kg (C :2) & & & & & 1.18 \\
\hline 23 & $\begin{array}{l}\text { Production efficiency (A } \\
\text { C) }\end{array}$ & & & & & \\
\hline 24. & $\begin{array}{l}\text { Revenue profitability (20 } \\
\text { A) x 100 }\end{array}$ & & & & & \\
\hline
\end{tabular}

Source: Authors' calculation based on data from Mičić, 2012

From Table 10 it is visible that breeding sows-piglets on farm B has the gain from 271,198 EUR, efficiency is 1.18 and revenue profitability is $15.07 \%$.

Table 11 shows achieved economic indicators in fattened pigs on farm B in 2012.

Table 11. Achieved economic indicators in fattened pigs on farm B

\begin{tabular}{|c|c|c|c|c|c|c|c|}
\hline 1. & $\begin{array}{l}\text { Production year: } \\
2012\end{array}$ & Amount & \multicolumn{3}{|c|}{ Conversion rate of feed in $\mathrm{kg}$ : } & 3.21 & $\mathrm{~kg} /$ growth \\
\hline 2. & $\begin{array}{l}\text { Fattening period: } \\
\text { Jan-Dec }\end{array}$ & $\begin{array}{c}\text { Unit of } \\
\text { Measure }\end{array}$ & \multicolumn{3}{|c|}{ Mortality rate of fattened: } & $2.0 \%$ & \\
\hline 3. & $\begin{array}{l}\text { Number of pigs } \\
\text { put for fattening: }\end{array}$ & 30,600 & livestock & & & & \\
\hline 4. & $\begin{array}{l}\text { Average weight } \\
\text { of a fattened pig: }\end{array}$ & 100 & $\begin{array}{c}\mathrm{kg} / \\
\text { livestock }\end{array}$ & & & & \\
\hline 5. & $\begin{array}{l}\text { Length of } \\
\text { fattening: }\end{array}$ & 98 & Days & & & & \\
\hline I & Revenue & - & $\begin{array}{c}\text { Unit of } \\
\text { measure }\end{array}$ & Price & $\begin{array}{c}\text { Unit of } \\
\text { measure }\end{array}$ & $\begin{array}{c}\text { Amount } \\
\text { total }\end{array}$ & $\begin{array}{c}\text { Amount } € / \\
\text { livestock }\end{array}$ \\
\hline 6. & $\begin{array}{l}\text { Fattened pigs( } \\
4 \times 6)\end{array}$ & 30,000 & livestock & 1,58 & $\mathrm{EUR} / \mathrm{kg}$ & $4,740,000.00$ & $\begin{array}{c}1,158.00 \\
\text { EUR }\end{array}$ \\
\hline 7. & Manure & 15,000 & $\mathrm{t}$ & 4,00 & $\mathrm{EUR} / \mathrm{t}$ & $60,000.00$ & 2.00 EUR \\
\hline 8. & $\begin{array}{l}\text { Subventions per } \\
\text { pig }\end{array}$ & 30,000 & livestock & 8,70 & $\begin{array}{c}\mathrm{EUR} / \\
\text { livestock }\end{array}$ & $261,000.00$ & 8,70 EUR \\
\hline A) & $\begin{array}{l}\text { Total revenue (1 } \\
\text { do } 8)\end{array}$ & - & & & & $5,061,000.00$ & $\begin{array}{c}168.70 \\
\text { EUR }\end{array}$ \\
\hline II & Expenses & - & & & & & \\
\hline
\end{tabular}




\begin{tabular}{|c|c|c|c|c|c|c|c|}
\hline 9. & $\begin{array}{l}\text { Piglets (average/ } \\
\text { pig) }\end{array}$ & 25.0 & $\begin{array}{c}\mathrm{kg} / \\
\text { livestock }\end{array}$ & & & & \\
\hline 10. & Piglets $(3 \times 9)$ & 765,000 & $\begin{array}{c}\mathrm{kg} / \\
\text { livestock }\end{array}$ & 2,04 & $\mathrm{EUR} / \mathrm{kg}$ & $1,560,600.00$ & 52.02 EUR \\
\hline 11. & \multicolumn{2}{|c|}{$\begin{array}{l}\text { Farm is has the mixture } \\
\text { according to pricelist }\end{array}$} & & & & & \\
\hline 12. & \multicolumn{2}{|c|}{$\begin{array}{l}\text { TS1 (from } 25-60 \mathrm{~kg} \text { ) } 2.35 \mathrm{~kg} / \\
\text { dayx46days x30,000 pigs }\end{array}$} & & 0,28 & $\mathrm{EUR} / \mathrm{kg}$ & $908,040.00$ & 30.27 EUR \\
\hline 13. & \multicolumn{2}{|c|}{$\begin{array}{l}\text { TS2 (from } 60-100 \mathrm{~kg} \text { ) } 2.55 \mathrm{~kg} / \\
\text { dayx } 52 \text { days x } 30,000 \text { pigs }\end{array}$} & & 0,26 & $\mathrm{EUR} / \mathrm{kg}$ & $1,034,280.00$ & 34.48 EUR \\
\hline 14. & $\begin{array}{l}\text { Average daily per } \\
\text { livestock }\end{array}$ & 2.46 & $\mathrm{~kg} / \mathrm{EUR}$ & & & & \\
\hline 15. & \begin{tabular}{|l|} 
Mechanical \\
work $(\mathbf{6} x \mathbf{x})$ \\
\end{tabular} & - & $\mathrm{kg} / \mathrm{EUR}$ & 1,4967 & $\mathrm{EUR} / \mathrm{kg}$ & $44,901.00$ & $1.50 \mathrm{EUR}$ \\
\hline 16. & $\begin{array}{l}\text { Water per } \\
\text { livestock }(16 \times 5) \\
x \text { 6:1.000 }\end{array}$ & 10 & $\mathrm{~L} /$ day & 1,15 & $\mathrm{EUR} / \mathrm{m}^{3}$ & $33,810.00$ & 1.12 EUR \\
\hline 17. & \multicolumn{2}{|c|}{$\begin{array}{l}\text { Veterinary services and } \\
\text { medicine }(6 x \text { 17) }\end{array}$} & & 0,80 & $\begin{array}{c}\text { EUR/ } \\
\text { livestock }\end{array}$ & $24,000.00$ & 0.80 EUR \\
\hline 18. & \multicolumn{2}{|c|}{ Human labor(4x6) $x 18$} & $\mathrm{~kg} / \mathrm{EUR}$ & 0,18 & $\begin{array}{c}\mathrm{EUR} / \\
\text { livestock }\end{array}$ & $540,000.00$ & 18.00 EUR \\
\hline 19. & \multicolumn{2}{|c|}{ Indirect costs (6 $x$ 19) } & $\mathrm{kg} / \mathrm{EUR}$ & 1,00 & $\begin{array}{c}\text { EUR/ } \\
\text { livestock }\end{array}$ & $30,000.00$ & 1.00 EUR \\
\hline 20. & \multicolumn{3}{|c|}{$\begin{array}{l}\text { Amortization of facilities and equipment } \\
(6 x \text { 20) }\end{array}$} & 3,53 & $\begin{array}{c}\text { EUR/ } \\
\text { livestock }\end{array}$ & $105,900.00$ & 3.53 EUR \\
\hline B) & \multicolumn{3}{|l|}{ Total costs(9 to 20) } & & & $4,281,531.00$ & $\begin{array}{c}142.72 \\
\text { EUR }\end{array}$ \\
\hline III & \multicolumn{3}{|l|}{ GAIN/LOSS } & & & & \\
\hline 21. & \multicolumn{3}{|c|}{ On farm with incentive $(\boldsymbol{A}-\boldsymbol{B})$} & & & $779,469.00$ & 25.98 EUR \\
\hline 22. & \multicolumn{3}{|c|}{ Cost per $\operatorname{kg} B:(4 x 6)$} & & & 1.42 & \\
\hline 23. & \multicolumn{3}{|c|}{ Production efficiency $(\boldsymbol{A}: \boldsymbol{B})$} & & & 1.18 & \\
\hline 24. & \multicolumn{3}{|c|}{ Revenue profitability $(21:$ A) $\times 100$} & & $\%$ & 15.40 & \\
\hline
\end{tabular}

Source: Authors'calculation based on data from Mičić, 2012

From Table 11 we can see that total achieved gain for 30,000 fattened pigs is $779,469.00$ EUR, production efficiency 1.18 and revenue profitability is $15.40 \%$.

Table 12 shows total achieved economic indicators in pig fattening on farm B in 2012.

Table 12. Total achieved economic indicators in pig fattening on farm B

\begin{tabular}{|c|l|c|c|c|c|c|c|c|}
\hline No. & $\begin{array}{l}\text { Production } \\
\text { year: }\end{array}$ & 2012 & Amount & Amount & $\begin{array}{c}\text { Unit of } \\
\text { measure }\end{array}$ & Price & $\begin{array}{c}\text { Unit of } \\
\text { measure }\end{array}$ & Amount EUR \\
\hline I & REVENUE & Livestock & & & & & & \\
\hline 2. & Manure(sow) & 1,500 & 500 & 750,000 & $\mathrm{~kg}$ & 0.01 & $€ / \mathrm{kg}$ & $7,500.00 \mathrm{EUR}$ \\
\hline 3. & Fattened pigs & 30,000 & 100 & $3,000,000$ & $\mathrm{~kg}$ & 1.58 & $€ / \mathrm{kg}$ & $4,740,000.00 \mathrm{EUR}$ \\
\hline 4. & $\begin{array}{l}\text { Manure } \\
\text { fattened pig) }\end{array}$ & 30,000 & 0.5 & 15,000 & $\mathrm{t}$ & 4.00 & $€ / \mathrm{t}$ & $60,000.00 \mathrm{EUR}$ \\
\hline
\end{tabular}




\begin{tabular}{|c|c|c|c|c|c|c|}
\hline 5. & Subventions & 30,000 & livestock & 8.70 & $\begin{array}{c}€ / \\
\text { livestock }\end{array}$ & $261,000,00 \mathrm{EUR}$ \\
\hline A) & Total revenue (1 to 5) & & & & & 5,068,500,00EUR \\
\hline II & EXPENSES & & & & & \\
\hline 6. & Fattened pigs & 30,000 & livestock & & $€$ & $4,281,531,00 \mathrm{EUR}$ \\
\hline В) & Total expenses & & & & & $4,281,531,00 \mathrm{EUR}$ \\
\hline III & GAIN/LOSS & & & & & \\
\hline 8. & Total with incentive $(\mathrm{A}-\mathrm{B})$ & & & & & $786,969.00 \mathrm{EUR}$ \\
\hline 9. & Production efficiency $(\mathrm{A}: \mathrm{B})$ & & & & & 1.18 \\
\hline 10. & Revenue profitability $(8: \mathrm{A}) \times 100$ & & & & $\%$ & 15.53 \\
\hline
\end{tabular}

Source: Authors'calculation based on data from Mičić, 2012

From Table 12 we can see that total realized gain on farm B was 786,969.00€, production efficiency 1.18 and revenue profitability $15.53 \%$.

\section{Production efficiency of fattened pigs on both farms in 2012}

In order to research production efficiency in fattening pigs, two farms from Serbia and Kosovo and Metohija were taken into consideration since they have the necessary conditions for such production. We researched farm A which has 40 fattened pigs and farm B which has 30,000 livestock. Average entrance weight of piglets for fattening was $25 \mathrm{~kg}$ and achieved exit weight was $100 \mathrm{~kg}$. On farm B average fattening period of pigs was 98 days with the daily gain of $0.76 \mathrm{~kg} /$ livestock/day, while on farm A it lasted 87 days, with an average daily growth of $0.86 \mathrm{~kg} /$ livestock $/$ day. Our results are in accordance with the results stated (Vidović et al., 2012) in the performance test of pure breeds of pigs, landrace and Yorkshire. Calculation of income includes only the revenue from selling pigs while potential revenue from pig meat wasn't taken into consideration (Table 13).

Table 13. Economic indicators of pork meat production - pork meat side on farm A and $\mathrm{B}$ in 2012

\begin{tabular}{|c|l|c|c|c|}
\hline I & Indicator & $\begin{array}{c}\text { Unit of } \\
\text { measure }\end{array}$ & FarmA/ & FarmB/ \\
\hline A. & Fresh pork meat sides & livestock & $\mathbf{4 0 . 0 0}$ & $\mathbf{3 0 , 0 0 0 . 0 0}$ \\
\hline B. & $\begin{array}{l}\text { Livestock weight of live pig } \\
\text { weight }\end{array}$ & $\mathbf{k g}$ & $\mathbf{1 0 0 . 0 0}$ & $\mathbf{1 0 0 . 0 0}$ \\
\hline 1. & Total weight, pig (A x B ) & $\mathbf{k g}$ & $4,000.00$ & $3,000,000.00$ \\
\hline 2. & Pork sides/livestock & $\%$ & 79.00 & 78.00 \\
\hline 3. & Total pork/side kg (1 x 2) : 100 & $\mathbf{k g}$ & $3,160.00$ & $2,340,000.00$ \\
\hline 4. & Price of pork sides & $€ / \mathbf{k g}$ & 2.82 & 2.82 \\
\hline V. & Total revenue $(\mathbf{3} \mathbf{x} \mathbf{4})$ & $€$ & $8,911.20$ & $6,598,800.00$ \\
\hline II & EXPENSES & & & \\
\hline 5. & Price of the slaughter service & $\begin{array}{c}€ / \\
\text { livestock }\end{array}$ & 8.70 & 8.70 \\
\hline
\end{tabular}




\begin{tabular}{|c|l|c|c|c|}
\hline 6. & Direct costs of live pig weight & $€$ & $3,480.00$ & $4,281,531.00$ \\
\hline 7. & $\begin{array}{l}\text { Total pig slaughter service }(\mathrm{Ax} \\
5)\end{array}$ & $€$ & 348.00 & $261,000.00$ \\
\hline G. & Total expenses( 6 + 7) & $€$ & $\mathbf{3 , 8 2 8 . 0 0}$ & $\mathbf{4 , 5 4 2 , 5 3 1 . 0 0}$ \\
\hline III & GAIN/LOSS & $€$ & & \\
\hline $\mathbf{8 .}$ & Pork sides from farms (V - G) & $€$ & $\mathbf{5 , 0 8 3 . 2 0}$ & $\mathbf{2 , 0 5 6 , 2 6 9 . 0 0}$ \\
\hline $\mathbf{9 .}$ & Pork side livestock/EUR (8: A) & $€$ & 127.08 & 68.54 \\
\hline $\mathbf{1 0 .}$ & Pork side price kg (G:3) & $€$ & 1.21 & 1.94 \\
\hline $\mathbf{1 1 .}$ & $\begin{array}{l}\text { Meat production efficiency (V } \\
: \text { G) }\end{array}$ & & 2.33 & 1.45 \\
\hline $\mathbf{1 2 .}$ & $\begin{array}{l}\text { Revenue profitability }(8: \mathrm{V}) \mathrm{x} \\
100\end{array}$ & $\%$ & 57.04 & 31.16 \\
\hline
\end{tabular}

Source: Authors' calculation based on data from Mičić, 2012

From the shown data it can be seen that pork meat side price on farm A is $1.21 \mathrm{EUR} /$ $\mathrm{kg}$ and that this production has the efficiency of 2.33 and revenue profitability of 57.04 $\%$, while on farm B pork meat side price is $1.94 \mathrm{EUR} / \mathrm{kg}$ and that this production has the efficiency of 1.45 and revenue profitability of $31.16 \%$. It can further be seen that on both farms the calculation of slaughter expenses, cooling and processing of sides amounts to 8.70 EUR per fattened pig.

Calculation was conducted in accordance with the achieved yield of slaughtered pigs, value of fattened pig with the mass of $100 \mathrm{~kg}$ with the price of $87.00 \mathrm{EUR}+8.70 \mathrm{EUR}$ slaughter expenses, which amounts to 85.70 EUR on farm A.

Slaughtered pigs have amounted to $79 \mathrm{~kg}$ of cooled side x 2.82 EUR price of a side, giving the value of 222.78 EUR, and when we take the price of pork meat (side) 95.70 EUR, we het the gain per livestock of $127.08 \mathrm{EUR} / \mathrm{kg}$. It can be easily concluded that the price of a cooled side is $132.79 \%$ bigger than the price of a fattened pig, which doesn't represent an usual relation on the market. Farm A achieved gain in the amount of 5,083.20 EUR.

A calculation was also conducted of farm B in accordance with the yield of slaughtered pigs and the value of a fattened pig with the weight of $100 \mathrm{~kg}$ is $142 \mathrm{EUR}+$ slaughter costs 8.7 EUR which amounts to 150.70 EUR, and since cooled pork meat side weighing $78 \mathrm{~kg}$ with the price of 2.82 EUR per $\mathrm{kg}$, the value of the livestock (side) is 219.96 EUR.

Slaughtering pigs they obtained pork meat sides with uniform mass, an average of 78 $\mathrm{kg}$ per livestock. Farm B realized a profit in the amount of 2,056,047 EUR.

It can easily be concluded that the price of a cooled side is $45.96 \%$ bigger than the price of a fattened pig.

We came to new scientific findings in the paper on the practical application of quality feeding in fattening pigs. Advantages of such a method of raising pigs was examined and the scientific contribution to promoting development of producing quality pork 
meat, for which Serbia with its pig raising tradition has great geographic-ecological potentials, especially in its agricultural and livestock production. As a final conclusion of the research it can be recommended to pig breeding farms to organize production groups, cooperatives, clusters and francizes so they could more easily open pig raisingrepro-center and perfect their products so they could be placed on the market faster.

\section{Conclusion}

Based on the analysis of the state in agricultural-food production, specifically pork meat, we came to a conclusion when the approach in this type of production is in question. Another argument in the request for determining fattened pig price on the slaughter line is the given quality according to the share of meat, which is shown in the research on farm A, that it's best to close the entire production cycle from a field to fork. We primarily think on the market of fattened pigs, piglets and pork meat in Serbia in 2012. After the research, we answered a few very important questions in the paper, which are: the price level is not such that it stimulates pig breeders and the price influences production level. One of the reasons the there is a reduction and stoppage of slaughtering of big industries in the Republic of Serbia (facilities in Sombor, Subotica and Čoka). There is an expansion of big private slaughterhouses at the same time, which are far more flexible the industries, therefore their production is far more efficient. Pork meat consumption cyclically reduces due to relatively high prices and a decline in life standard. An increase in price of this products leads to lower demand and meat consumption. The abovementioned indicator shows very significant economic effects of the stated technology in feeding pigs, high strategic efficiency in production development. Pork meat production on both farms is economically justified and development of intensive production of goods can be expected in farm conditions in Serbia.

\section{Literature}

1. Andrić, J. (1998): Troškovii kalkulacije u poljoprivrednoj proizvodnji, Poljoprivredni fakultet - Zemun, Beograd; Council Regulation (EEC) No 3220/84 of 13 November 1984 determiningthe Community scale forgrading pig carcass, 1984. Official Journal ofthe European Communities No L 301, 20/11/1984, No. $1-3$.

2. Džinić, N., Petrović, Lj., Tomović, V., Manojlović, D., Timanović, S., Trišić-Ilić, S., Mrdjanov, J. (2003): Uticaj višerasnog ukrštanja i godišnjeg doba na kvalitet polutki i mesa svinja, Savremena poljoprivreda, Vol. 52, No. 3-4, pp. 335-337.

3. Džinić, N., Petrović, Lj.,Tomović, V., Manojlović, D., Timanović, S., Vidanrić, D., Kurjakov, N. (2004): Ocena kvaliteta polutki i mesa svinja rasa veliki jorkšir $i$ švedski ladras. Biotehnologija u stočarstvu, Vol. 20, No. 1-2, pp. 67-73.

4. Fisher, A.V., Green, D.M., Whittemore, C.T., Wood, J.D., Schofield, C.P. (2003): Growth of carcass components and its relation with conformation in pigs of three types, Meat Science, Vol. 65, No. 1, pp. 639-650. 
5. Kosovac, O., Josipović, S., Živković, B., Radović, Č., Marinkov, G., Tomašević, D. (2007): Comparable presentation of carcass and meat quality of different pig genotypes using modern evaluation methods,. $2^{\text {nd }}$ International Congress on animal husbandry ,New perspectives and challenges of sustainable livestock farming", Belgrade, Serbia, Biotechnology In Animal Husbandry, Vol. 23, No. 5-6, pp 291-301.

6. Jovanović, M., Tica, N. (1998): Kalkulacije u poljoprivredi, Poljoprivredni fakultet, Novi Sad.

7. Jukna, V., Jukna, C. (2005): The comparable estimation of meat quality of pigs breeds and their combinations in Lithuania, Biotechnology in Animal Husbandry, Vol. 21, No. 5-6, pp. 175-179

8. Manojlović, D., Petrović, Lj.,Džinić, N., Kurjakov, N. (1999): Kvalitet trupa i mesa svinja-osnova kvaliteta proizvoda,Monografija: Tehnologijaproizvodnje i kvalitet konzervi od mesa u komadima, No. 67-90, Tehnološkifakultet u NovomSadu.

9. Orović, D., LjubanovićRalević, I., Anokić, A.(2015): Assessment ofbusiness efficiency of agriculturalholdings withdifferent productions, Economics of Agriculture Belgrade Serbia, Vol. 62, No. 3, pp. 781-799.

10. Pravilnik o načinuostvarivanjapravanapodstiscaje u stočarstvupokošnicipčelaPublished in „Official gazete of the RS”, no. 60 /13 from 10. July 2013.

11. Rahelić, S. (1984): Uzgoj svinje i meso, Školskaknjiga, Zagreb.

12. Šegrt, S., Kolarski, I. (2015): Uslovi egzistencije preduzeća, Oditor, Belgrade, no. 11 , pp. $32-41$

13. Tešanović, D. (1969): Uticaj starosti pri prašenju na finansiski rezultat proizvodnje, Ekonomika poljoprivrede, No. 7 - 8, Belgrade.

14. Timanović, S., Vidanić, D., Kurjakov, N. (2003): Ocena kvaliteta polutki i mesa svinja rasa veliki jorkšir i švedski landras, Biotehnologija u stočarstvu Vol. 19, No. 5-6, p. 500

15. Tomović, V., Petrović, Lj.,Džinić, N.,Manojlović,D.,Timanović, S., Trišić-Ilić, S. (2005): Meat yield in primal cuts and sides of pigs determinedby partial dissection, International $5^{\text {3rd }}$ meat industryconference "New integral approach to meat and products safety", Vrnjačkabanja, I-15, No. 31-34.

16. Vidović, V., Krnjaić, J., Lukač, D., Višnjić, V., Stupar, M. (2012): Growthintensity ofthe giltsfertile breedpigsinthe Nucleusfarm,Biotech, Anim. Hus., Vol. 28, No. 4, pp. 787-796.

17. Živković, D., Perunović, M. (2012): Poznavanje mesa, praktikum, Poljoprivredni fakultet, Univerzitetu Beogradu.

18. Okanović, Đ., Zekić, V., Petrović, L. S., Tomović, V., Džinić, N. R. (2006): Ekonomičnost proizvodnj esvinjskog mesa u polutkama,Tehnologija mesa, Vol. 47, No. 5-6, pp. 237-241.

19. Zekić, V., Tomović, V., Milić, D., Vidović, V., Lukač, D. (2013): Ekonomska obeležja proizvodnje svinja rase landras i mangulica, Letopis naučnih radova Poljoprivrednog fakulteta, Vol. 37, No. 1, pp. 191-199

20. Zekić, V., Tomović, V., Milić, D., Lukač, D. (2012): Poređenje ekonomskih 
obeležja tova svinja mangulica i svinja rase jorkšir,Ekonomika poljoprivrede, Vol. 59, No. 4, pp. 649-656.

21. Zekić, V., OKANOVIĆ, Đ. (2007): Prudence of fatty pigs production, I International Congress: „FOOD TECHNOLOGY,QUALITY AND SAFETY“, XI Symposium NODA: ,Technology, quality andsafety in pork production and meat Processing“, Proceedings No. 33-37. 


\section{TROŠKOVI POLJOPRIVREDNIH GAZDINSTAVA (FARMA, POLJ. PREDUZEĆA, SELJAČKO GAZDINSTVO) SA JEDNAKIM MOGUĆNOSTIMA PROIZVODNJE}

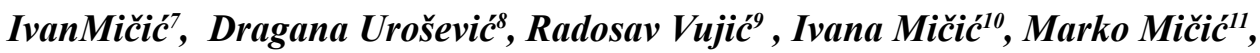 Marija Mičić ${ }^{12}$}

\section{Rezime}

Predmet istraživanja u radu je analiza stanja $i$ osnovnih problema u farmskoj proizvodnji svinjskog mesa, kao i ekonomska analiza tova svinja. Istraživanja su obuhvatila konkretne studije slučaja na porodičnom poljoprivrednom gazdinstvu, svinjogojskoj farmi u Gračanici, u centralnom delu Kosova i Metohije i na zadružnom poljoprivrednom gazdinstvu, svinjogojsku farmu u Žitorađi u Topličkom okrugu. U periodu u 2012. godini praćen je obim i primenjena tehnologija proizvodnje tovnih svinja na obe farme i analizirani su dobijeni ekonomski rezultati. U posmatranom periodu utvrđeno je da na privatnoj farmi ukupan broj tovljenika iznosi 40 grla, dok na zadružnoj farmi, broj tovljenika iznosi 30.000 grla. Proizvedeni tovljenik na privatno jfarmikošta $87 €$, $i$ svinjsko meso u polutki $1,16 € / \mathrm{kg}$. Na zadružnoj farmi cena tovljenika iznosi $142 €$, i svinjsko meso u polutki 1,94 €/kg. Prosečna masa tovljenika na obe farme je $100 \mathrm{~kg}$, dok je udeo svinjskih polutki varirao od 78\%-79\%.

Ključne reči: Proizvodnja tovljenika, svinjskog mesa - polutki, cena, kvalitet, ekonomski rezultati.

7 Mr Ivan Mičić, doktorand, Univerzitet u Beogradu, Poljoprivredni fakultet, Nemanjina ulica br. 6, 11080 Zemun, Srbija, Telefon:+381 1126153 15,E-mail:divanlav@gmail.com

8 Master Dragana Urošević, Univerzitet u Beogradu, Poljoprivredni fakultet, Nemanjina ulica br. 6, 11080 Zemun, Srbija, Telefon: +381 6388149 35, E-mail: dragana. urosevic91@yahoo.com

9 Dr Radosav Vujić, Poljoprivredno stručna i savetodavna službaValjevo, Birčaninova br. 128 A, 14000 Valjevo,Srbija, Telefon: +381648435458 E-mail: pssvaljevo@mts.rs

10 Master Ivana Mičić, doktorand, Ekonomski fakultet, Univerzitet u Nišu, Trg Kralja Aleksandra Ujedinitelja 11, 18000 Nišu, Srbija, Telefon: +381 63233 603, E-mail: (ivancica@gmail.com)

11 Marko Mičić, Dipl. ecc, Univerzitet u Nišu, Ekonomski fakultet ,Trg Kralja Aleksandra Ujedinitelja 11, 18000 Nišu, Srbija,Telefon: +381 63680 040, E-mail: (markomicic89@, gmail.com)

12 Master Marija Mičić, doktorand, Univerzitet u Nišu, Tehnološki fakultet u Leskovcu, Bulevar Oslobođenja 124, 16000 Leskovac, Srbija, Telefon: +381 62867 45 98, E-mail: (marija84micic@gmail.com) 